\title{
THE LAWS OF SIMILITUDE AND CRACK PROPAGATION
}

\author{
Anthony G. Atkins and Robert M. Caddell \\ Department of Mechanical Engineering, University of Michigan, Ann Arbor, \\ Michigan 48104, U.S.A.
}

(Received 21 September 1973)

\begin{abstract}
Summary-The mechanics of cracking follow the laws of similitude in an odd sense. As a result, crack load-oxternal displacement-crack extension data are not usually nondimensionalized. It follows that a new "group" should be used (analogous to the Reynolds, Froude or Cauchy numbers) when scaling ship ice-breaking resistance from tests of models in ice-towing-tanks.
\end{abstract}

\section{NOTATION}

$K$ stress intensity factor

$\sigma$ applied stress

$a$ half of crack length

$R$ fracture toughness

$E$ elastic modulus

$\lambda$ linear scaling factor

$p, m$ subscripts denoting prototype and model

$F$ applied force

$t, w, h$ symbols denoting thickness, width and height of a specimen

$\varepsilon$ strain

$u$ displacement

$A$ crack area

$\mathscr{E}$ dimensionless velocity parameter

$\dot{c}$ crack velocity

$\dot{u}$ crosshead velocity

$L$ characteristic length

$F_{I}$ inertia force

$M$ mass

$\ddot{s}$ acceleration

$F_{\mathrm{v}}$ viscous force

$\mu$ viscosity

$\partial \dot{s} / \partial y \quad$ velocity gradient

$F_{\mathrm{G}}$ gravity force

$g$ gravitational acceleration

$\rho$ density

$\tau$ time scaling factor

$\nu$ kinematic viscosity

$v$ velocity

$R_{n}$ Reynolds number

$F_{\mathbf{n}}$ Froude number

$F_{\mathrm{e}}$ elastic force

$C_{\mathrm{n}}$ Cauchy number

$c_{0}$ sonic velocity

$M_{n}$ Mach number

$I_{\mathrm{n}}$ ice number 


\section{INTRODUCTION}

The ENERGY approach of Gurney in refs. (1)-(3) to the mechanics of cracking and the stability of crack propagation is followed below in developing the concepts presented in this paper. Gurney's method of attack is related to "fracture mechanics" in the same way that Castigliano strain energy theorems for elastic displacements relate to elasticity calculations based upon integration of strain expressions. ${ }^{4}$

The question of the applicability of laboratory toughness data to the design of large scale structures is discussed. It is common practice to employ laboratory fracture toughness data in formulae such as $K=\sigma \sqrt{ } a$ or $R=\pi \sigma^{2} a / E$, to determine the design stress, $\sigma$, in the structure being considered. It seems to be less well known however that the stress so calculated is lower than the stress to cause cracking in a geometrically similar, but smaller, structure. In fact, $\sigma_{p}=\sigma_{m} \lambda^{-1}$, see ref. (1), where the subscripts $\mathrm{p}$ and $\mathrm{m}$ refer to prototype (large structure) and model respectively, and $\lambda$ is the linear scaling factor which relates a characteristic prototype dimension to the analogous model dimension, $(\lambda>1)$.

Additionally, it has been implied ${ }^{5}$ that cracking velocities in the prototype are faster than those in the model by the factor $\lambda$. This is important with regard to strain rate effects and crack stabilities in small and large structures (cf. the use of small testpieces inside large pressure vessels to keep track of stress corrosion).

An important consequence of this lack of similitude is that typical crack load-external displacement-crack extension data are not usually normalized in the same sense that load-extension curves are converted to stress-strain curves, for example, irrespective of testpiece size. Although the 'equivalent energy' normalization (e.g. ref. 6) may be shown to follow correct scaling laws, its common application to irreversible cases of post-yield fracture of materials with high $K / \sigma^{2}$ should be questioned.

We concentrate rather on a significant finding concerning the interpretation of data obtained with models breaking ice, and its application to large scale situations. The behavior of model ships in ice towing tanks and model piers etc. as affected by ice forces come to mind. In particular it is shown that a new non-dimensional group emerges, akin to the Reynolds, Froude and Cauchy numbers, which must be satisfied if scaling from model to prototype is to be done correctly.

\section{SIMILITUDE AND CRACKING}

Consider a model cracked structure and a prototype cracked structure, see Fig. 1. Typical stresses and strains in the model are given by

$$
\left.\begin{array}{c}
\sigma_{\mathrm{m}}=F_{\mathrm{m}} / t_{\mathrm{m}} w_{\mathrm{m}}, \quad \varepsilon_{\mathrm{m}}=\frac{u_{\mathrm{m}}}{h_{\mathrm{m}}}, \quad E_{\mathrm{m}}=\frac{F_{\mathrm{m}} h_{\mathrm{p}}}{t_{\mathrm{m}} w_{\mathrm{m}} u_{\mathrm{m}}}, \\
\sigma_{\mathrm{p}}=F_{\mathrm{p}} / t_{\mathrm{p}} w_{\mathrm{p}}, \quad \varepsilon_{\mathrm{p}}=\frac{u_{\mathrm{p}}}{h_{\mathrm{p}}}, \quad E_{\mathbf{p}}=\frac{F_{\mathrm{p}} h_{\mathrm{p}}}{t_{\mathrm{p}} w_{\mathrm{p}} u_{\mathrm{p}}},
\end{array}\right\}
$$

where the Young's moduli of model and prototype are allowed to be different. 

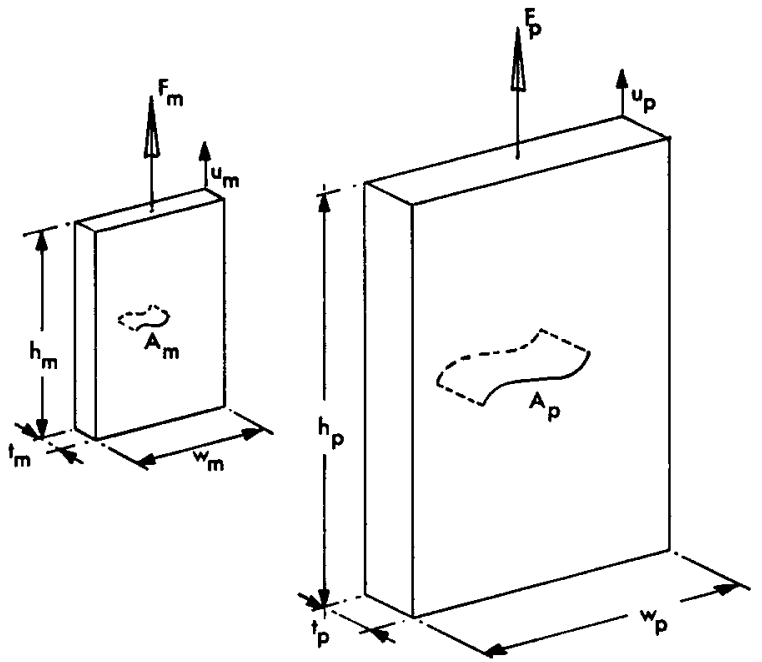

$$
\frac{h_{p}}{h_{m}}=\frac{w_{p}}{w_{m}}=\frac{t_{p}}{t_{m}}=\lambda
$$

FIG. 1. Nomenclature for geometrically similar cracked pieces.

Considering the compliances $(u / F)$ of model and prototype, it follows that

$$
\frac{u_{\mathrm{p}}}{F_{\mathrm{p}}}=\frac{l}{\lambda} \frac{u_{\mathrm{m}}}{F_{\mathrm{m}}} \frac{E_{\mathrm{m}}}{E_{\mathrm{p}}}
$$

and that the rates of change of compliance with respect to crack area are

$$
\frac{\mathrm{d}}{\mathrm{d} A_{\mathrm{p}}}\left(u_{\mathrm{p}} / F_{\mathrm{p}}\right)=\frac{1}{\lambda^{2}} \frac{\mathrm{d}}{\mathrm{d} A_{\mathrm{m}}}\left(u_{\mathrm{p}} / F_{\mathrm{p}}\right)=\frac{1}{\lambda^{3}} \frac{E_{\mathrm{m}}}{E_{\mathrm{p}}} \frac{\mathrm{d}}{\mathrm{d} A_{\mathrm{m}}}\left(u_{\mathrm{m}} / F_{\mathrm{m}}\right) .
$$

One version of the fundamental equation relating cracking force, external displacement and crack extension is, ref. 1 ,

$$
F^{2}=2 R / \frac{\mathrm{d}}{\mathrm{d} A}\left(\frac{u}{F}\right)
$$

Consequently, when inserted into the cracking force equation we have

$$
F_{\mathrm{p}}^{2}=2 R_{\mathrm{p}} / \frac{\mathrm{d}}{\mathrm{d} A_{\mathrm{p}}}\left(\frac{u_{\mathrm{p}}}{F_{\mathrm{p}}}\right)=2 R_{\mathrm{p}} / \frac{1}{\lambda^{3}} \frac{E_{\mathrm{m}}}{E_{\mathrm{p}}} \frac{\mathrm{d}}{\mathrm{d} A_{\mathrm{m}}}\left(\frac{u_{\mathrm{m}}}{F_{\mathrm{m}}}\right)
$$

and also

$$
F_{\mathrm{m}}^{2}=2 R_{\mathrm{m}} / \frac{\mathrm{d} A_{\mathrm{m}}}{\mathrm{d}}\left(\frac{u_{\mathrm{m}}}{F_{\mathrm{m}}}\right) \text { and } F_{\mathrm{p}}=\lambda\left(\frac{E_{\mathrm{p}} R_{\mathrm{p}}}{E_{\mathrm{m}} R_{\mathrm{m}}}\right)^{\frac{1}{\mathrm{~m}}} F_{\mathrm{m}}=\lambda F_{\mathrm{m}} \frac{K_{\mathrm{p}}}{K_{\mathrm{m}}},
$$

where $K=\sqrt{ }(E R)$ is the stress intensity factor for the material.

Thus, if $K_{\mathrm{p}}=K_{\mathrm{m}}$, the cracking load in the large piece is bigger than that in the small piece by $\lambda$. Since the areas, over which these forces act, differ by $\lambda^{2}$ it follows that

$$
\sigma_{\mathrm{p}}=\frac{1}{\sqrt{ } \lambda} \sigma_{\mathrm{m}}
$$

Hence the stresses to propagate cracks are smaller in larger pieces and, as pointed out by Gurney, ${ }^{1}$ cracking can occur before yielding in large structures whereas the same material can display general yielding before cracking in small structures.

Also, since

$$
u_{\mathrm{p}}=u_{\mathrm{m}} \frac{1}{\lambda} \frac{F_{\mathrm{p}} E_{\mathrm{m}}}{F_{\mathrm{m}} E_{\mathrm{p}}}
$$


we have

$$
u_{\mathrm{p}}=\lambda^{\frac{1}{2}}\left(\frac{K_{\mathrm{p}} E_{\mathrm{m}}}{K_{\mathrm{m}} E_{\mathrm{p}}}\right) u_{\mathrm{m}},
$$

i.e. for equal $K, E$ between model and prototype

$$
u_{\mathrm{p}}=\lambda^{\frac{1}{2}} u_{\mathrm{m}} .
$$

Hence the displacements in the prototype are bigger than those in the model only by $\lambda^{\frac{1}{2}}$.

When constant $R$ loci are plotted in the $(F, u)$ plane according to equation (4), we obtain diagrams such as in Fig. 2, the precise shape of the loci depending on the geometry of the cracked structure. For linearly elastic systems, different crack lengths (areas) are represented by a series of radial lines emanating from the origin each reflecting the compliance of the cracked structure as it is influenced by the different crack areas. An important interpretation of equation (4), is that sector areas such as $O A_{1} A_{2}$ represent the work done in extending the crack from area $A_{1}$ to area $A_{2}$, i.e. $R\left(A_{2}-A_{1}\right)$. When cracks are stable and well behaved, this allows a ready means of evaluating $R$, see refs. (1) $-(3)$ and $(5)$.
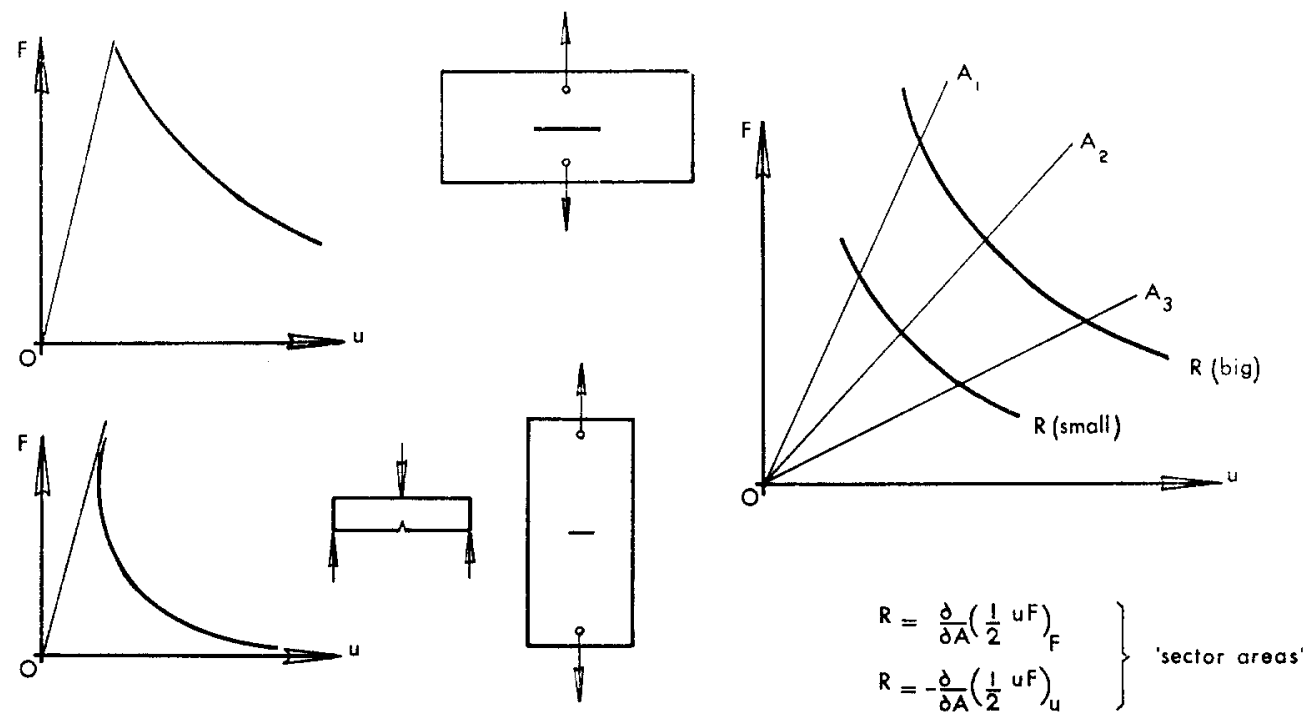

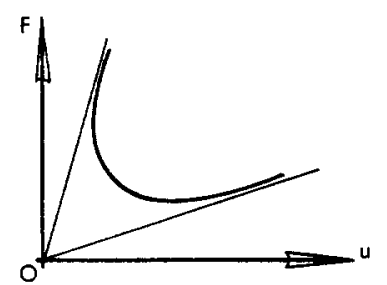

(a)

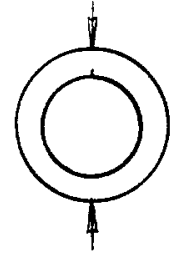

FIG. 2. Various constant fracture toughness loci for different shape cracked structures (a) and (b).

Load-extension data from uncracked pieces are usually interpreted to give stresses and strains that allow determination of Young's moduli and yield strengths, etc. In geometrically similar pieces, the load increases as $\lambda^{2}$ and the extension by $\lambda$, which when reduced to $\sigma$ and $\varepsilon$, lead to a single curve. The area under this curve is the work done/unit volume; in a large piece the work done is $\lambda^{2}$ (small load) $\times \lambda$ (small extension) $=\lambda^{3}$ (work on small piecej.

However, since the volume of the larger piece is bigger than the smaller by a factor of $\lambda^{3}$ the work done/unit volume is constant. 
Consider the analogous treatment of cracking data. Are there "master curves', (constant $R$ loci) for a given shape testpiece, for which sector areas represent the work done in cracking? Evidently for pieces of the same material if the loads are divided by $\lambda^{\text {t }}$ and the extensions by $\lambda^{\prime}$ (see equations (6) and (10)) we get "universal" $R$ loci. The radial lines from the origin representing constant crack areas are normalized at the same time, since

$$
\frac{u_{\mathrm{p}} / \lambda^{\underline{z}}}{F_{\mathrm{p}} / \lambda^{i}}=\lambda \frac{u_{\mathrm{p}}}{F_{\mathrm{p}}}
$$

and since also for the same material,

Regarding work done,

$$
\frac{u_{\mathrm{p}}}{F_{\mathrm{p}}}=\frac{1}{\lambda} \frac{u_{\mathrm{m}}}{F_{\mathrm{m}}} \text { and } \frac{u_{\mathrm{p}} / \lambda^{1}}{\bar{F}_{\mathrm{p}} / \lambda^{\frac{3}{3}}}=\frac{u_{\mathrm{m}}}{F_{\mathrm{m}}}
$$

$$
\frac{F_{\mathrm{p}}}{\lambda^{3}} \frac{u_{\mathrm{p}}}{\lambda^{\underline{t}}}=\frac{1}{\lambda^{2}} F_{\mathrm{p}} u_{\mathrm{p}}=F_{\mathrm{m}} u_{\mathrm{m}}
$$

$F_{\mathrm{p}} u_{\mathrm{p}}$ "feeds" an area $\lambda^{2}$ bigger than does $F_{\mathrm{m}} u_{\mathrm{m}}$, so the fracture toughness work is equivalent for both cases. These effects are shown schematically in Fig. 3.

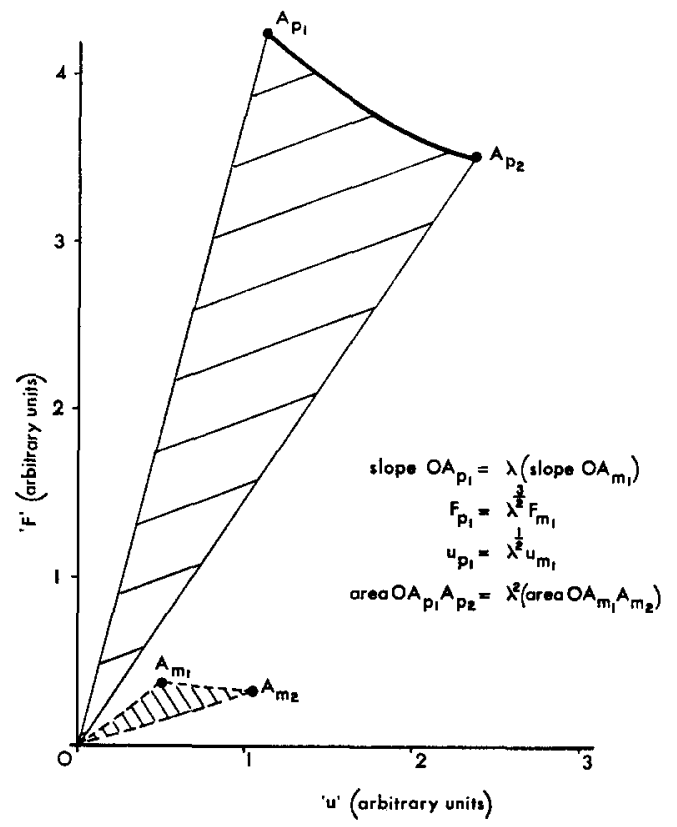

FIG. 3. Method of normalizing crack load-crack extension-external displacement data; $\lambda$ equals 5 in this scaled drawing.

However, the present authors have shown ${ }^{5}$ that crack velocities are faster in larger structures. The ratio of crack velocity to driving (crosshead) velocity depends upon a parameter, $\mathscr{E}$, as follows:

$$
\frac{\dot{c}}{\dot{u}}=\frac{E \sqrt{ } L}{K} \mathscr{E}
$$

Since $\mathscr{E}$ is constant for geometrically similar testpieces, it follows that prototype velocity ratios are bigger than model velocity ratios by the factor $\lambda$. Consequently, if $K$ is rate dependent, the same $K$ loci may not be picked up by normalizing load-extension data in the manner just described from different size specimens. 


\section{APPLICATION TO ICE-TANK TOWING STUDIES}

It is well known that in conventional towing tank experiments, with models of prototype ships or structures, it is generally impossible to arrange complete dynamic similarity, i.e. the independent force ratios concerning inertia forces, viscous forces and gravity forces in model and prototype do not have the same scaling factors."

The reasons are as follows. Inertia forces are given by $F_{I}=M \ddot{s}$, viscous forces by $F_{v}=\mu A(\partial \dot{s} / \partial y)$, gravity forces by $F_{G}=M g$ and, for completeness, elasticity forces by $F_{\mathrm{e}}=\sigma A=E \varepsilon A$. Thus it may be shown that ${ }^{8}$

$$
\frac{\left(F_{\mathrm{I}}\right)_{\mathrm{p}}}{\left(F_{\mathrm{I}}\right)_{\mathrm{m}}}=\frac{\rho_{\mathrm{p}} \lambda^{4}}{\rho_{\mathrm{m}} \tau^{2}}, \quad \frac{\left(F_{\mathrm{v}}\right)_{\mathrm{p}}}{\left(F_{\mathrm{v}}\right)_{\mathrm{m}}}=\frac{\nu_{\mathrm{p}}}{\nu_{\mathrm{m}}} \frac{\rho_{\mathrm{p}}}{\rho_{\mathrm{m}}} \frac{\lambda^{2}}{\tau^{2}}, \quad \frac{\left(F_{\mathrm{G}}\right)_{\mathrm{p}}}{\left(F_{\mathrm{G}}\right)_{\mathrm{m}}}=\frac{\rho_{\mathrm{p}}}{\rho_{\mathrm{m}}} \lambda^{3}, \quad \frac{\left(F_{\mathrm{e}}\right)_{\mathrm{p}}}{\left(F_{\mathrm{e}}\right)_{\mathrm{m}}}=\frac{E_{\mathrm{p}}}{E_{\mathrm{m}}} \lambda^{2}
$$

Consequently, to satisfy the same scaling factor for inertia forces and viscous (friction) forces,

$$
\frac{\rho_{\mathrm{p}}}{\rho_{\mathrm{m}}} \frac{\lambda^{4}}{\tau^{2}}=\frac{\nu_{\mathrm{p}}}{\nu_{\mathrm{m}}} \frac{\rho_{\mathrm{p}}}{\rho_{\mathrm{m}}} \frac{\lambda^{2}}{\tau^{2}}
$$

so that the time scale between model and prototype is

$$
\tau=\frac{\nu_{\mathrm{m}}}{\nu_{\mathrm{p}}} \lambda^{2}
$$

The velocity ratio between model and prototype is

Therefore, we have

$$
\frac{v_{\mathrm{p}}}{v_{\mathrm{m}}}=\frac{\lambda}{\tau}=\frac{\nu_{\mathrm{p}}}{\nu_{\mathrm{m}}} \frac{1}{\lambda}=\frac{\nu_{\mathrm{p}}}{\nu_{\mathrm{m}}} \frac{L_{\mathrm{m}}}{L_{\mathrm{p}}}
$$

$$
\frac{v_{\mathrm{p}} L_{\mathrm{p}}}{\nu_{\mathrm{p}}}=\frac{v_{\mathrm{m}} L_{\mathrm{m}}}{\nu_{\mathrm{m}}}=\mathbf{R}_{\mathrm{n}} \quad \text { (Reynolds number). }
$$

Likewise we can show that for equal scaling factors between inertia and gravity (wave making) forces

$$
\frac{v_{\mathrm{p}}}{\sqrt{L_{\mathbf{p}}}}=\frac{v_{\mathrm{m}}}{\sqrt{L_{\mathrm{m}}}} \quad \text { (speed : length ratio) }
$$

or

$$
\frac{v_{\mathrm{p}}}{\sqrt{\left(g L_{\mathrm{p}}\right)}}=\frac{v_{\mathrm{m}}}{\sqrt{\left(g L_{\mathrm{m}}\right)}}=F_{\mathrm{n}} \quad \text { (Froude number) }
$$

Again, for simultaneous satisfaction of inertia and elastic forces

$$
v_{\mathrm{p}}\left(\frac{\rho_{\mathrm{p}}}{\bar{E}_{\mathrm{p}}}\right)^{\frac{1}{2}}=v_{\mathrm{m}}\left(\frac{\rho_{\mathrm{m}}}{E_{\mathrm{m}}}\right)^{\frac{1}{2}}=C_{\mathrm{n}} \text { (Cauchy number) }
$$

or since $(E / \rho)^{\ddagger}=c_{0}$ (the sonic velocity), we have

$$
\frac{v_{\mathrm{p}}}{c_{\mathrm{op}}}=\frac{v_{\mathrm{m}}}{c_{0 \mathrm{~m}}}=\mathbf{M}_{\mathrm{n}} \quad \text { (Mach number). }
$$

Since tank fluids are incompressible for all practical purposes, $C_{\mathrm{n}}$ is neglected in open water towing studies, and $\mathrm{M}_{\mathrm{n}}$ appears more in wind tunnel work. Regarding $F_{\mathrm{n}}$ and $\mathrm{R}_{\mathrm{n}}$, complete dynamic similitude is impossible unless a tank fluid cen be found for which $\nu_{\mathrm{p}} / \nu_{\mathrm{m}}=\lambda \mathrm{I}$. It is customary to scale according to Froude's law and correct the test results by independent experimental "plate" data based upon Reynolds' law.

When dealing with towing basins covered in ice to model the ice-breaking capabilities of prototype ships or ice-affected structures, for example, the situation is complicated beyond the above considerations since the ice-breaking force ratios must satisfy some scaling law. Some workers argue that the Cauchy number for the ice sheet is the relevant scaling parameter, but since we have shown that cracking forces should not be identified continuum elastic forces, $C_{\mathrm{n}}$ is not appropriate. Rather a new number has to be satisfied, based upon the force relationship $F_{\mathrm{p}}=\lambda\left(K_{\mathrm{p}} / K_{\mathrm{m}}\right) F_{\mathrm{m}}$ (equation (6)), as follows: 
In order to satisfy the same scaling law for inertia forces and cracking forces, we have from arguments analogous to those presented earlier,

i.e.

$$
\frac{\rho_{\mathrm{p}}}{\rho_{\mathrm{m}}} \frac{\lambda^{4}}{\tau^{2}}=\lambda\left(\frac{K_{\mathrm{p}}}{K_{\mathrm{m}}}\right)
$$

and

$$
\tau=\left(\frac{\rho_{\mathrm{p}}}{\rho_{\mathrm{m}}} \lambda \frac{K_{\mathrm{m}}}{K_{\mathrm{p}}}\right)^{\mathbf{z}}
$$

so that

$$
\frac{v_{\mathrm{p}}}{v_{\mathrm{m}}}=\frac{\lambda}{\tau}=\left(\frac{\rho_{\mathrm{m}} K_{\mathrm{p}}}{\rho_{\mathrm{p}} K_{\mathrm{m}}}\right)^{\frac{1}{\lambda^{z}}}
$$

$$
v_{\mathrm{p}}\left(\frac{\rho_{\mathrm{p}} L_{\mathrm{p}}^{\mathbf{z}}}{K_{\mathrm{p}}}\right)^{\frac{1}{2}}=v_{\mathrm{m}}\left(\frac{\rho_{\mathrm{m}} L_{\mathrm{m}}}{K_{\mathrm{m}}}\right)^{\mathbf{t}}
$$

or

$$
\frac{v_{\mathrm{p}}^{2} \rho_{\mathrm{p}} L_{\mathrm{p}}^{\mathbf{k}}}{K_{\mathrm{p}}}=\frac{v_{\mathrm{m}}^{2} \rho_{\mathrm{m}} L_{\mathrm{m}}^{\mathbf{m}}}{K_{\mathrm{m}}}=I_{\mathrm{n}} \quad \text { (new "ice" number). }
$$

Notice that $I_{\mathrm{n}}=C_{\mathrm{n}}^{2}(E L / R)^{4}$, so that $I_{\mathrm{n}}$ appears to be the Cauchy number generalized to include the effects of a "defective" (cracked) continuum.

Scaling laws for ice tank work are ill understood at present and the techniques used to translate from model to prototype are quasi-empirical. The foregoing analysis gives a new scaling law for the ice fracture part of the resistance of a ship to passage through ice or, conversely, to the forces on dams, etc. caused by ice jams. It is customary to weaken the strength of tank ice relative to sea ice or lake ice. This is accomplished by copiously salting the tank water to reduce the ice strength. Brine pockets in the frozen ice are the cause of weakening. It is known that the mechanical properties of such ice sheets markedly differ from "prototype" ice. Not only is the strength diminished, but the ice moduli are also altered, the tank ice being almost rubber-like in flexibility. The "rules" by which model ice "strength"* is diminished, at present are arbitrary. However, it can be shown" that by arranging the mechanical properties of model ice in a particular way, both Froude's law and the cracking law can be simultaneously satisfied. This allows model testing at the characteristically (slow) Froude speeds.

It may be argued that the brine pockets in model ice are not that much smaller than those in real ice, so that $A_{m} \neq\left(1 / \lambda^{2}\right) A_{\mathrm{p}}$. The mathematics leading to $I_{\mathrm{n}}$ are easily modified to accommodate this variation, which gives a slightly different expression for $I_{n}$, but trends just discussed are equally valid.

\section{CONCLUSIONS}

Cracking loads, cracking stresses, and crack velocities in geometrically similar pieces obey unusual scaling laws. The similarity concepts have been applied to the particular problem of scaling test data from ice model basins into full size situations (ships, piers, etc.). A new non-dimensional group has been proposed for similitude in ice cracking studies, which should shed light on the ill-understood problem of 'total' modelling of a ship's resistance to passage through ice.

\section{REFERENCES}

1. C. Gurney and J. Hunt, Proc. Roy. Soc. A297, 508 (1967).

2. C. Gurney and K. M. NGaN, Proc. Roy. Soc. A325, 207 (1971).

3. C. GURNeY and Y. W. MAI, Engnr. Fracture Mech. 4, 853 (1972).

* The "fracture strength" $\sigma$ is determined by in situ cantilever beam tests. Since strength ultimately has to do with $R$ or $K$ the use of $\sigma$ in scaling laws is to be discouraged. 
4. A. G. Atkins and R. M. CADdell, Engnr. Fracture Mech. (in press).

5. A. G. Atkins, R. M. Caddell, C. S. Lee and R. L. MoCarthy, Am. Soc. Mech. Engrs, Winter Meeting, New York (1972).

6. American Society for Testing and Materials, Special Technical Publication No. 536, Philadelphia (1973).

7. J. K. VenNard, Elementary Fluid Mechanics. Wiley, New York (1962).

8. I. SzABo, Einführung in die Technische Mechanik, Vol. 112. Springer, Berlin (1961).

9. A. G. Atkins, Advanced Arctic Marine Technology Conference (1973). 